\title{
Sinus Augmentation Utilizing Calcium Phosphosilicate Biomaterial Followed by Delayed Implant Rehabilitation: Radiological and Clinical Analyses
}

\author{
Rayapati Dilip Kumar, Honey Verma, NT Prashanth, ES Shobha
}

\begin{abstract}
The direct sinus lift approach is a well-established technique for implant placement in the deficient maxilla. The need to provide efficacious treatment to patients while minimizing procedure-associated morbidity lead to the introduction to bone substitutes as alternative to autogenous bone grafting for bone augmentation in the sinus.

Calcium phosphosilicate biomaterial is the form of morsels that have been recently introduced for use in oral surgery. The osteostimulative properties of this biomaterial makes it an excellent bone substitute for indications that have been traditionally treated with autogenous bone graft.

A case is presented where implant rehabilitation was planned in left maxillary posterior region with pre-existent significant pneumatization of the sinus. Under local anesthesia direct sinus lift technique was employed. The results were highly satisfactory showing regeneration up to approximately $13 \mathrm{~mm}$ of bone height that allowed successful implant placement of appropriately sized implant fixtures.

The present case-study is a proof-of-principle study of the efficacy of morsels in direct sinus lift. Use of morsels for sinus augmentation provides successful outcomes with minimal morbidity for the patient and significant decrease in chair-side time that would be required for harvest of an autograft.
\end{abstract}

Keywords: Sinus lift, Bone grafts, Dental implants, СBCT.

How to cite this article: Kumar RD, Verma $H$, Prashanth NT, Shobha ES. Sinus Augmentation Utilizing Calcium Phosphosilicate Biomaterial Followed by Delayed Implant Rehabilitation: Radiological and Clinical Analyses. Int J Oral Implantol Clin Res 2013;4(3):102-107.

Source of support: Nil

Conflict of interest: None declared

\section{INTRODUCTION}

The direct sinus lift approach was initially described by Boyne and James in 1980. Their rationale was to restore, or even augment the bone volume that was originally present below the sinus floor prior to pneumatization. ${ }^{1}$ Soon after the introduction of this technique several authors verified the feasibility of the lateral window approach for predictable implant placement in the posterior maxilla. ${ }^{2,3}$

Boyne and James advocated the use of autogenous bone marrow for the fill of the sinus cavity following sinus floor elevation. Autogenous bone harvesting is inevitably 45 associated with donor site morbidity. ${ }^{4,5}$ The need to provide efficacious treatment to patients while minimizing procedure-associated morbidity lead to the introduction to bone substitutes as alternative to autogenous bone grafting for bone augmentation in the sinus. ${ }^{6}$ Numerous techniques and a wide variety of bone graft substitutes have been introduced over the years for the purpose bone augmentation in oral surgical procedures including autografts, allografts, xenografts and alloplasts. ${ }^{4,7-10}$ The highest level of evidence is available to show that synthetic bone graft substitutes are at least as efficient, if not more, in comparison to $100 \%$ autogenous bone for bone augmentation in the sinus. ${ }^{11}$

New evidence-based concepts in sinus augmentation dictate the use of alloplastic biomaterials for improved results with minimization of morbidity during sinus augmentation. ${ }^{12}$ Calcium phosphosilicate (CPS) biomaterials have recently attracted much attention due to their osteostimulative properties. ${ }^{7,8}$ CPS biomaterials are osteostimulative are considered osteostimulative as they have been shown to undergo both physical and chemical dissolution when implanted. ${ }^{13,14}$ This is a result of an ion release mechanism that occurs between the biomaterial and body tissues resulting in in situ formation of hydroxycarbonite apatite (HCA) which remodels into bone.

The increased interest in the dental community for CPS biomaterials has been instigated by the broad range of biomaterial formulations that increase their ease of handling.

The aim of this case report was to present a case of direct sinus lift with the aid of a novel CPS alloplastic bone graft that resulted in significant bone formation in the sinus with minimum morbidity for the patient.

\section{CASE REPORT}

The patient, a 52-year-old female with noncontributory medical history, presented for implant placement in the upper left maxillary region. Clinical examination of the area revealed adequate ridge width for implant placement as well as adequate interarch distance for placement of an implant-supported restoration. CBCT was selected as the radiographic examination of choice due to the potential need for a bone augmentation procedure. ${ }^{15}$ Preoperative cone beam CT (CBCT) assessment revealed significant pneumatization of the sinus with residual bone height ranging from 4.1 to $5.4 \mathrm{~mm}$ (Fig. 1). The buccolingual dimensions of the ridge radiographically were adequate for placement of a standard diameter implant, as had been indicated during the preoperative clinical examination (Fig. 2). Thickening of the schneiderian membrane was also noted in several of the cross-sectional images that was consistent with history of chronic sinusitis. In preparation for implant treatment bone quality was visually assessed on 
Sinus Augmentation Utilizing Calcium Phosphosilicate Biomaterial Followed by Delayed Implant Rehabilitation
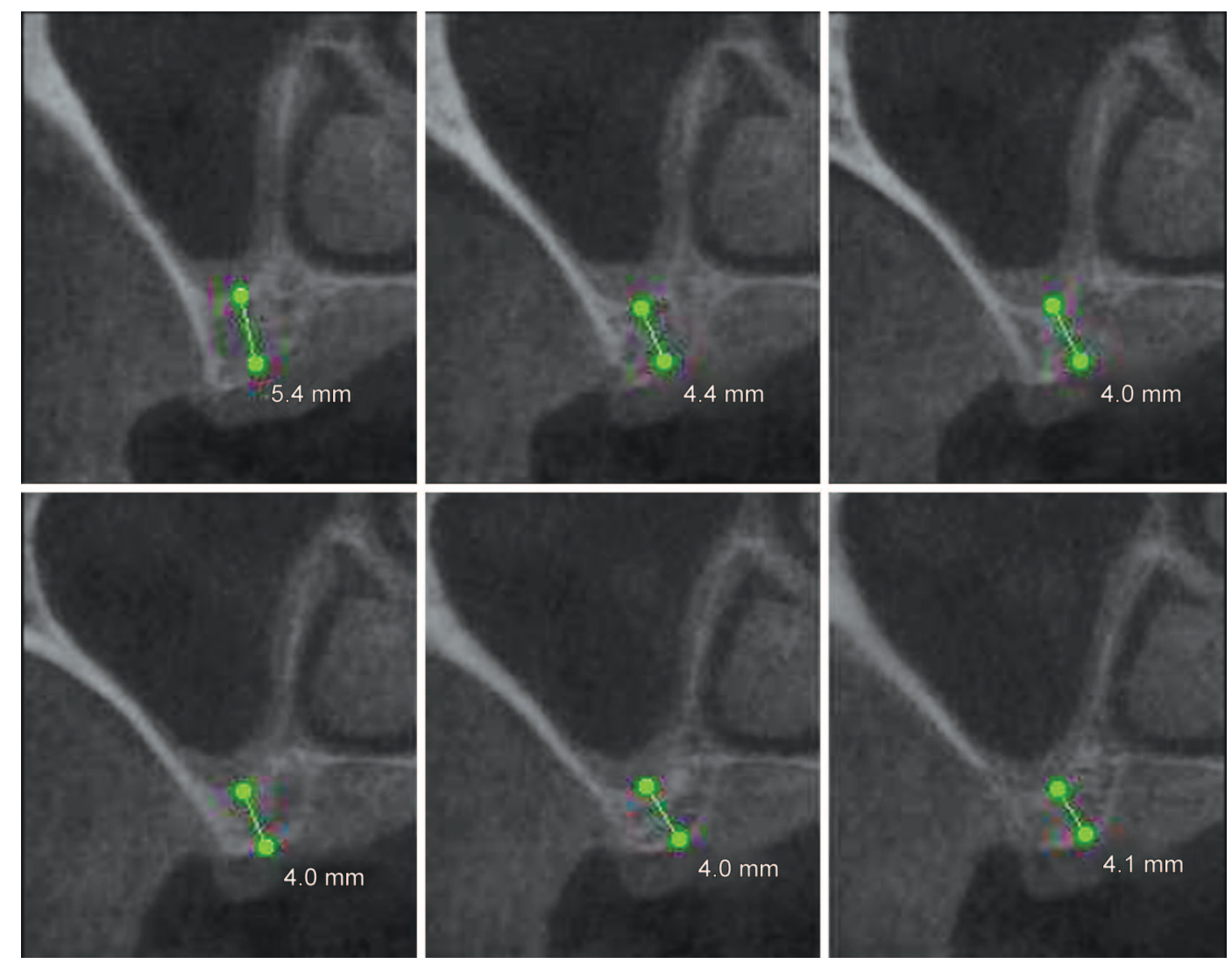

Fig. 1: Preoperative CBCT scans showing the measurement of bone in terms of height in edentulous left maxillary posterior region
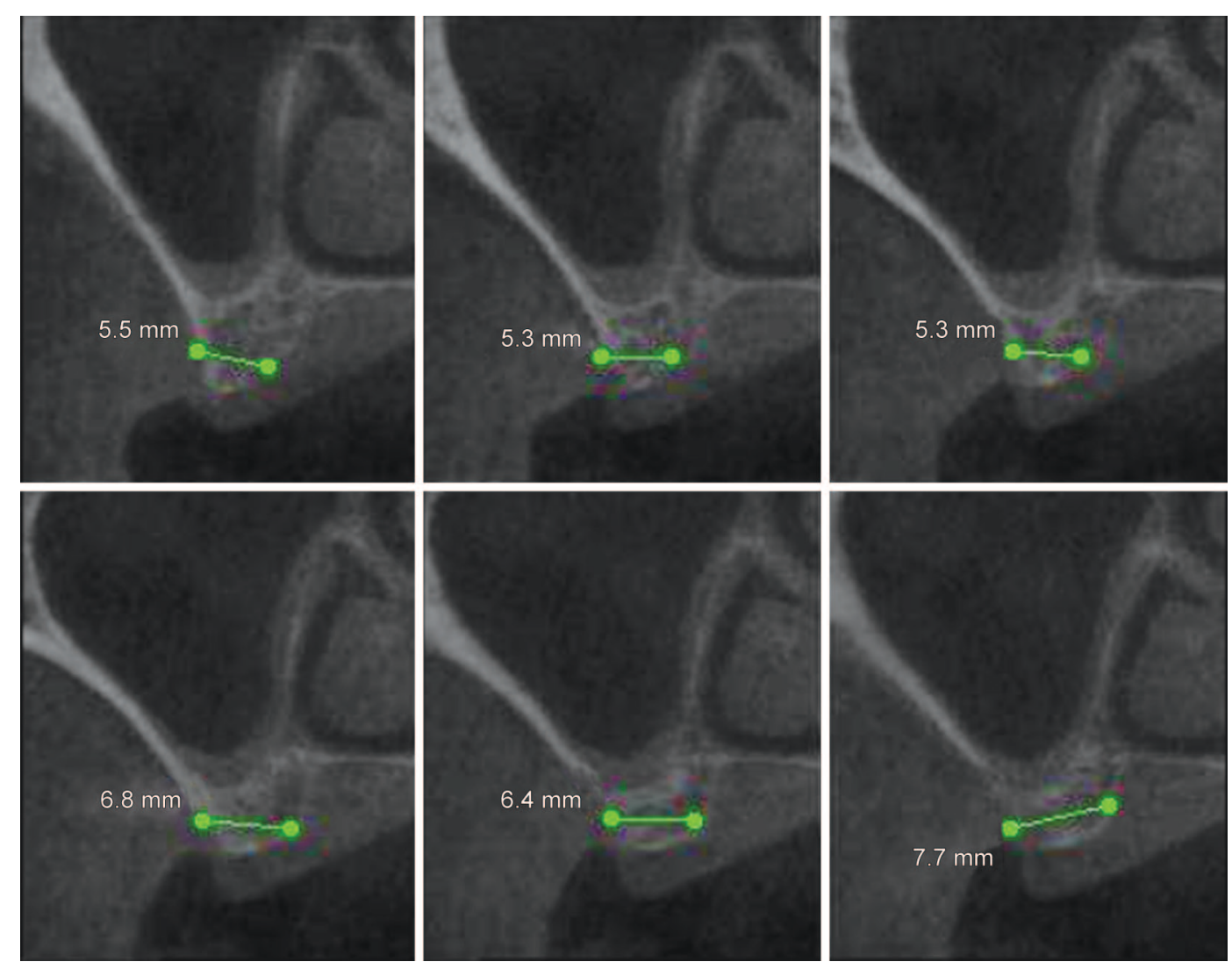

Fig. 2: Preoperative CBCT scans showing the measurement of bone in terms of width in edentulous left maxillary posterior region 
the CBCT. The wide porous structure of the medullary bone was indicative of grade IV bone quality. ${ }^{16}$

Due to the diminished preoperative bone height dimensions and the poor quality of the bone in the posterior maxilla, it was decided to proceed with direct sinus lift to achieve at least $10 \mathrm{~mm}$ of bone height and subsequent implant placement after 6 months of healing. The patient agreed with the proposed treatment plan and consented to treatment.

\section{Surgical Procedure (Sinus Augmentation)}

Following administration of local anesthesia the patient was instructed to rinse with $0.12 \%$ of chlorhexidine mouthwash preoperatively in order to reduce the microbial load. A crestal incision was traced extending from distal surface of the maxillary left canine posteriorly to the upper left maxillary tuberosity region. A mucoperiosteal flap was raised on the buccal to expose the anterior wall of maxillary sinus. Under copious irrigation with sterile saline, a stainless steel No. 4 round bur was employed to open a window of $1 \times 1 \mathrm{~cm}^{2}$ (postage stamp method) on the lateral wall of the sinus. The schniderian membrane was identified. Gently the sinus membrane was pushed superiorly using sinus instrument/ curettes, assuring no tear in the membrane (Fig. 3). A valsava method was utilized to verify the integrity of the membrane. The space that was created by the lift of membrane was grafted with $4 \mathrm{cc}$ of Novabone morsels (Novabone LLC, Alachua, Florida, USA) enriched with platelet rich plasma (Fig. 4). The consistency of the morsels following mixing with the platelet rich plasma enhanced the characteristics of this bone graft even further and allowed it to be placed in the sinus avoiding overcondensation (Fig. 5). Following verification of fill of the sinus cavity with the graft up to the desired height the flaps were approximated and passively secured with routine interrupted suturing technique (Fig. 6). The patient received appropriate antibiotic and analgesic regimen as well as postoperative instruction prior to release.

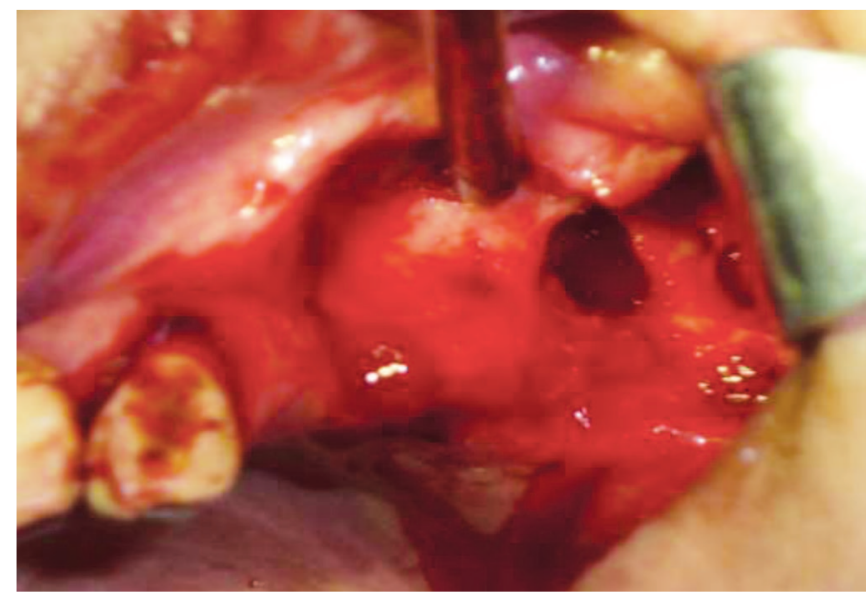

Fig. 3: Exposure of maxillary sinus floor on left side assuring no tear in the membrane

\section{RESULTS AND FOLLOW-UP}

The patient presented for a 2-week follow-up appointment with no subjective symptoms. Intraoral evaluation revealed good primary closure with almost complete epithelization of the incision wound. Minimal edema was noted intraoperatively with no signs of active inflammation.

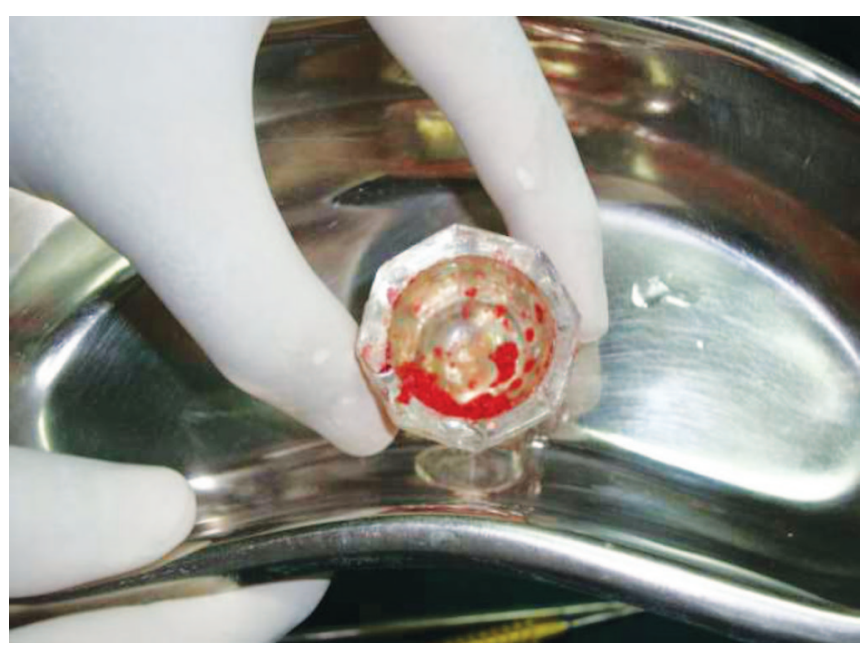

Fig. 4: Novabone morsels mixed with fresh blood of patient and platelet rich plasma

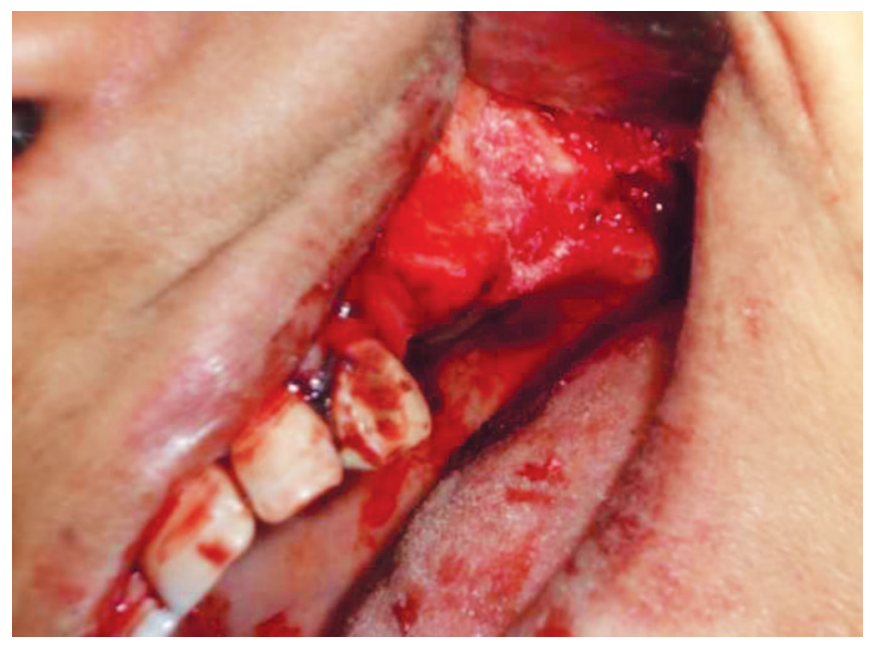

Fig. 5: Area grafted with the material

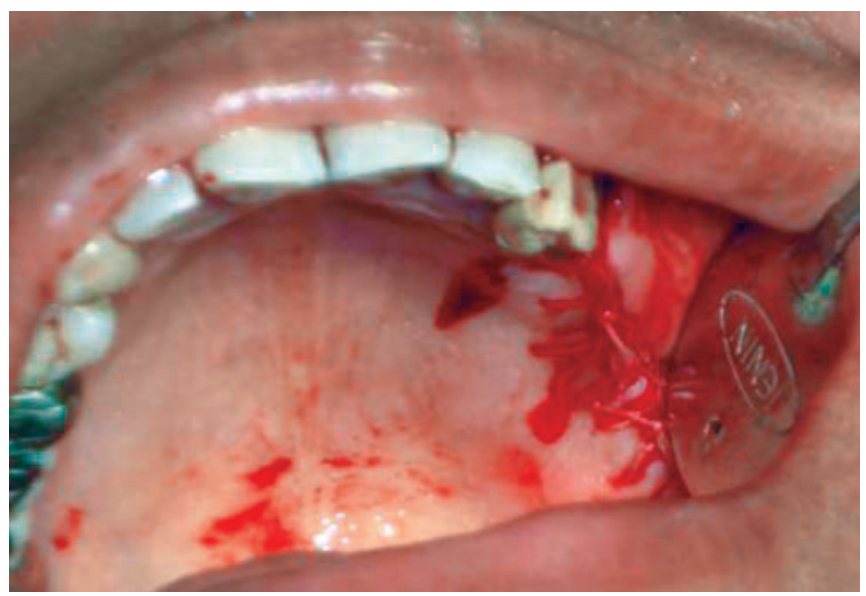

Fig. 6: Wound primary closure 


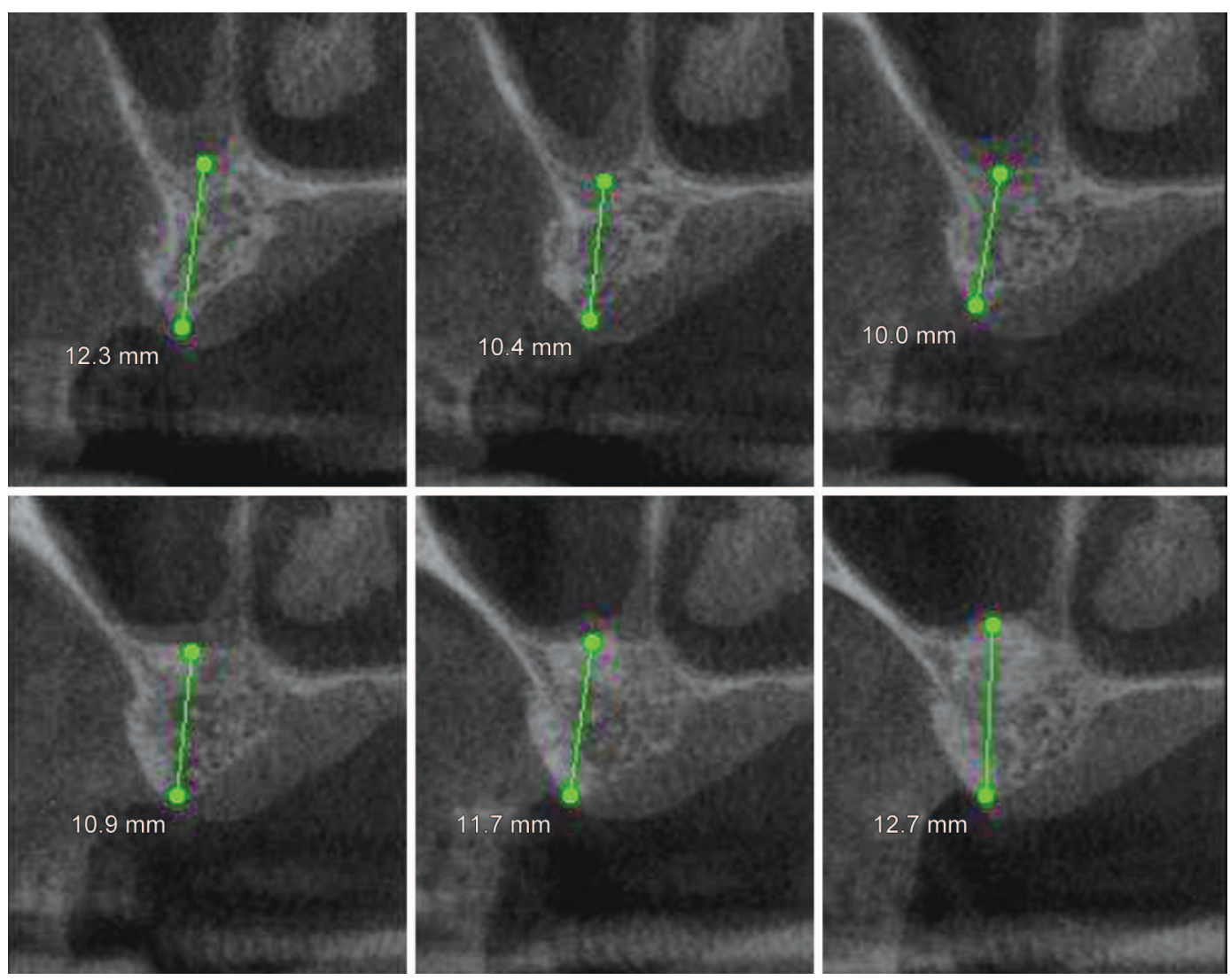

Fig. 7: Postoperative CBCT scan shows overall increase in quantity of bone. Bone height is increased to receive an appropriate size of implants
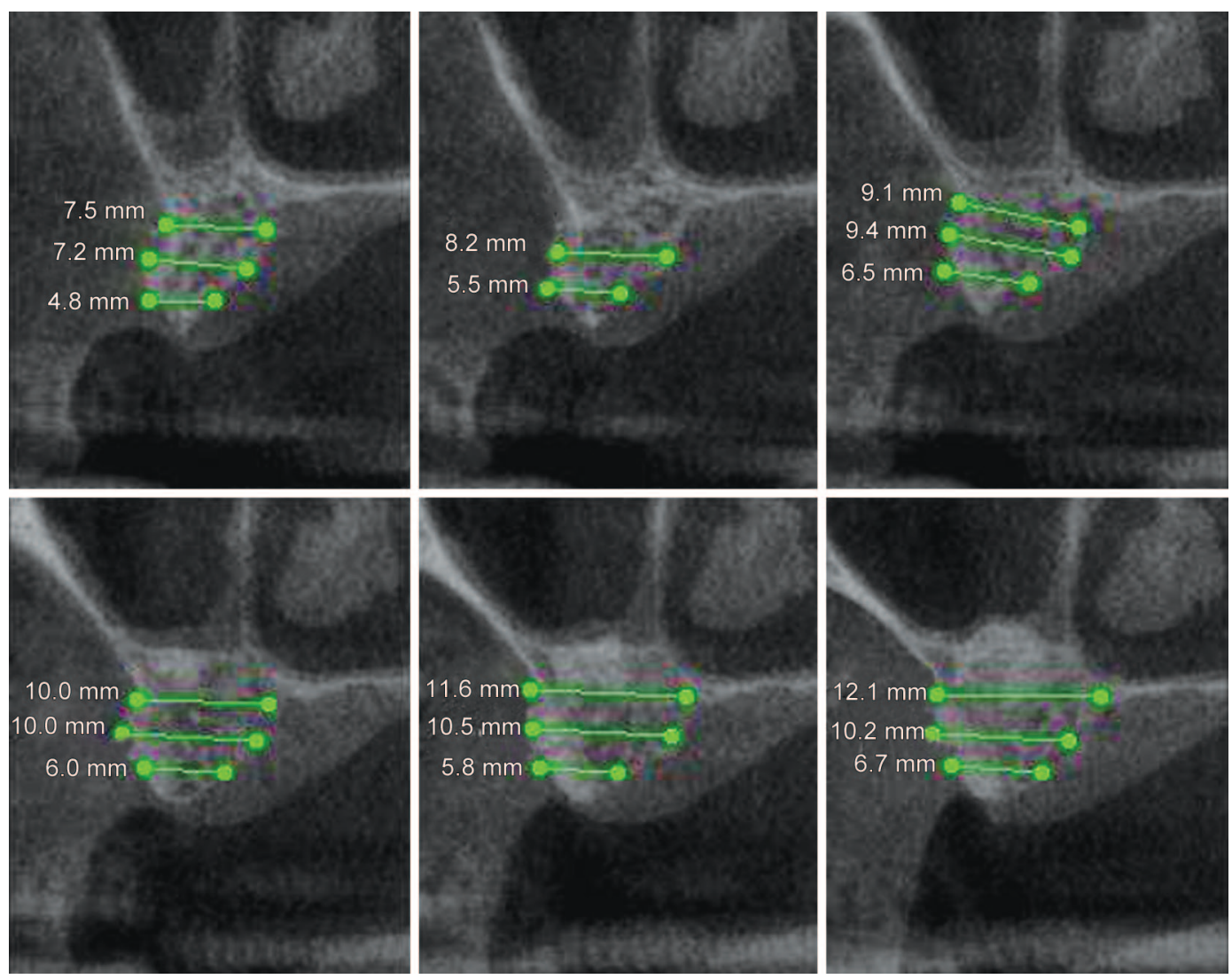

Fig. 8: Ridge dimensions were also favorably improved in a buccolingual dimension. Note the favorable bone quality of the regenerated bone 


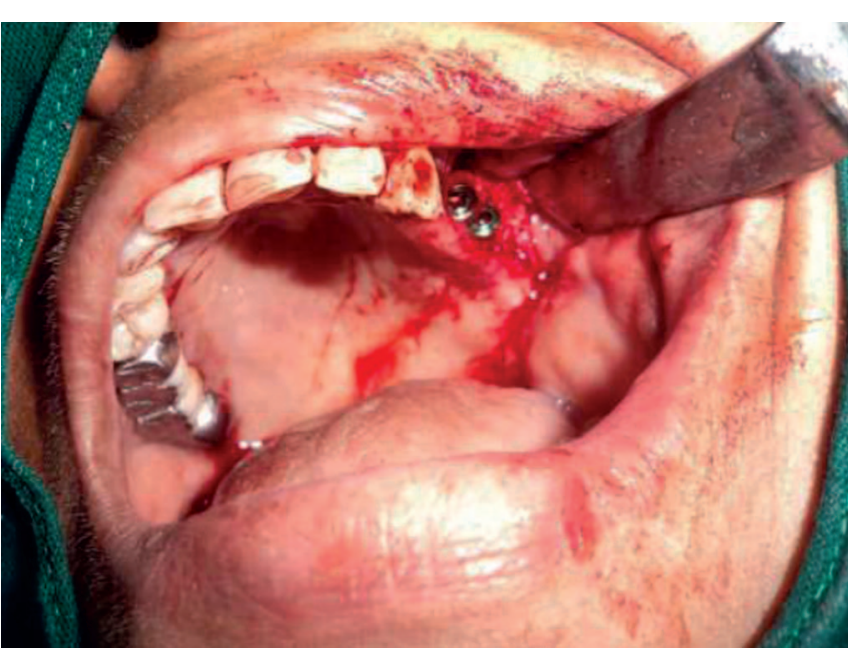

Fig. 9: Implants placed in the grafted region

At 3 months postoperatively, a CBCT was taken. The postoperative $\mathrm{CBCT}$ was compared with preoperative scan values to evaluate the improvement in bony contours following augmentation. It was found that approximately 5 to $7 \mathrm{~mm}$ of bone height gain was achieved (Fig. 7).

Up to $12.7 \mathrm{~mm}$ of grafted bone height were noted in the maxillary region with no site exhibiting less than $10 \mathrm{~mm}$ of height. Note the very nice trabecular pattern of the regenerated bone (Fig. 8).

The bone formed was adequate in terms of quantity and quality hence it was decided that 3 implants of appropriate size would be placed in the grafted region.

\section{Surgical Procedure (Implant Placement)}

At 6 months postgraft placement the site was re-entered with minimal flap reflection following standard implant surgery principles. Three implants were placed in the healed site according to standard drilling protocol with ample sterile saline irrigation. All three implants achieved optimal primary stability showing good bone quality regenerated in the surgical sites (Fig. 9).

\section{RESULTS AND FOLLOW-UP}

The patient was recalled regularly to evaluate healing in the area. The healing proceeded uneventfully and no complications were noted throughout the recall period.

A 3 months postoperative, radiographic examination (Fig. 10) shows good stability of the regenerated bone in the sinus and absence of radiolucency around the implants. The implants were successfully uncovered at this time and restored with porcelain-fused-to metal restorations. The patients reported significant increase in his masticatory

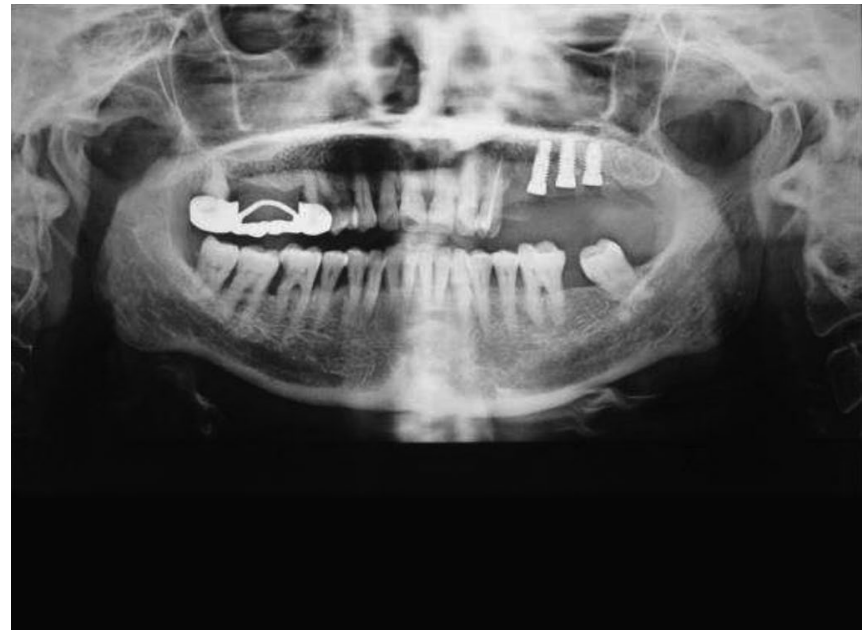

Fig. 10: Digital panoramic radiograph showing well-osseointegrated implants in the grafted region

function and was very pleased with the outcome. At the 6 months follow-up appointment the implants remained healthy and in function with no signs of complications.

\section{DISCUSSION}

In a 52 years old female, implant rehabilitation was planned in left maxillary posterior region. Preoperative CBCT as a preoperative radiological assessment aid showed pneumatization of left maxillary sinus, dipping toward edentulous ridge and offering minimum bone available for implant placement which indicated a sinus lift procedure to be carried prior to implant rehabilitation. Under local anesthesia direct sinus lift technique was employed. The results were highly satisfactory showing regeneration up to approximately $13 \mathrm{~mm}$ of bone height that allowed successful implant placement of appropriately sized implant fixtures.

The lateral window approach technique for sinus augmentation offers excellent visual control of the surgical site, particularly for reduced chair-side times and minimal morbidity mandate the use of alloplastic biomaterials. ${ }^{12}$

The introduction of morsels provides clinicians with an excellent alternative to autogenous bone grafting. Morsels are essentially comprised of calcium phosphosilicate (CPS). CPS biomaterials have been reported to exhibit promising results when used for bone augmentation and ridge preservation procedures. ${ }^{17-19}$ In addition to being osteoconductive this biomaterial has been characterized as osteostimulative by the FDA (FDA 510k 2006) due to its ability to stimulate osteoprogenitor cells to produce transforming growth factor, owing to the release of silicon. ${ }^{20}$ Bioactivity of CPS results from the chemical release of ionic dissolution products: silicon, sodium, calcium and phosphate and has shown to stimulate multiple generations of undifferentiated cells into osteoblasts. ${ }^{21}$ 
CPS has been successfully used in various osseous defects with no reported adverse events. ${ }^{17-19}$ The present case-study is a proof-of-principle study of the efficacy of morsels in direct sinus lift. Use of dental morsels for sinus augmentation provides successful outcomes with minimal morbidity for the patient and significant decrease in chairside time that would be required for harvest of an autograft.

\section{REFERENCES}

1. Boyne PJ, James RA. Grafting of the maxillary sinus floor with autogenous marrow and bone. J Oral Surg 1980;38(8):613-616.

2. Kent JN, Block MS. Simultaneous maxillary sinus floor bone grafting and placement of hydroxylapatite-coated implants. J Oral Maxillofac Surg 1989;47(3):238-242.

3. Mazor Z, Peleg M, Gross M. Sinus augmentation for single-tooth replacement in the posterior maxilla: a 3-year follow-up clinical report. Int J Oral Maxillofac Implants 1999;14(1):55-60.

4. Dahlin C, Johansson A. Iliac crest autogenous bone graft versus alloplastic graft and guided bone regeneration in the reconstruction of atrophic maxillae: a 5-year retrospective study on cost-effectiveness and clinical outcome. Clin Implant Dent Relat Res 2011;13(4):305-310.

5. Park JJ, Hershman SH, Kim YH. Updates in the use of bone grafts in the lumbar spine. Bull Hosp Jt Dis 2013;71(1):39-48.

6. Whittaker JM, James RA, Lozada J, Cordova C, GaRey DJ. Histological response and clinical evaluation of heterograft and allograft materials in the elevation of the maxillary sinus for the preparation of endosteal dental implant sites. Simultaneous sinus elevation and root form implantation: an eight-month autopsy report. J Oral Implantol 1989;15(2):141-144.

7. Kotsakis G, Chrepa V, Marcou N, Prasad H, Hinrichs J. Flapless alveolar ridge preservation utilizing the 'socket-plug' technique: clinical technique and review of the literature. J Oral Implantol 2012.

8. Mahesh L, Kotsakis G, Venkataraman N, Shukla S, Prasad H. Ridge preservation with the socket-plug technique utilizing an alloplastic putty bone substitute or a particulate xenograft: a histological pilot study. J Oral Implantol 2013.

9. Mahesh L, Salama MA, Kurtzman GM, Joachim FP. Socket grafting with calcium phosphosilicate alloplast putty: a histomorphometric evaluation. Compend Contin Educ Dent 2012;33(8):e109-115.

10. Wheeler SL. Sinus augmentation for dental implants: the use of alloplastic materials. J Oral Maxillofac Surg 1997;55(11): 1287-1293.

11. Del Fabbro M, Rosano G, Taschieri S. Implant survival rates after maxillary sinus augmentation. Eur J Oral Sci 2008;116(6): 497-506.

12. Wallace SS, Tarnow DP, Froum SJ, et al. Maxillary sinus elevation by lateral window approach: evolution of technology and technique. J Evid Based Dent Pract 2012;12(3 Suppl):161-171.

13. Tsigkou O, Hench LL, Boccaccini AR, Polak JM, Stevens MM. Enhanced differentiation and mineralization of human fetal osteoblasts on PDLLA containing Bioglass composite films in the absence of osteogenic supplements. J Biomed Mater Res A 2007;80(4):837-851.

14. Hench LL. The story of Bioglass. J Mater Sci Mater Med 2006;17(11):967-978.

15. Farman AG, Scarfe WC, Ahmad M. American academy of oral and maxillofacial radiology commentary: position papers in perspective. Oral Surg Oral Med Oral Pathol Oral Radiol Endod 2011;111(2):238.

16. Misch CE, Dietsh-Misch F, Hoar J, et al. A bone quality-based implant system: first year of prosthetic loading. J Oral Implantol 1999;25(3):185-197.

17. Mahesh L, Salama MA, Kurtzman GM, Joachim FP. Socket grafting with calcium phosphosilicate alloplast putty: a histomorphometric evaluation. Compendium Contin Educ Dent Sept 2012;33(8):109-115.

18. Kotsakis G, Chrepa V, Marcou N, Prasad H, Hinrichs J. Flapless alveolar ridge preservation utilizing the 'socket-plug' technique: clinical technique and review of the literature. J Oral Implantol 2012 Nov 12. [Epub ahead of print]

19. Kotsakis G, Chrepa V, Katta S. Practical application of the newly introduced natural bone regeneration (NBR) concept utilizing alloplastic putty. Int J Oral Imp Clin Res Sept-Dec 2011;2(3):145-149.

20. Price N, Bendall S, Frondoza C, et al. Human osteoblast-like cells (MG63) proliferate on a bioactive glass surface. J Biomed Mater Res 1997;37:394-400.

21. Xynos ID, Edgar AI, Buttery LDK, Hench LL. Polak JM. Geneexpression profiling of human osteoblasts following treatment with the ionic products of bioglass 45S5 dissolution. J Biomed Mater Res 2001 May;55(2):151-157.

\section{ABOUT THE AUTHORS}

\section{Rayapati Dilip Kumar}

Professor, Department of Oral and Maxillofacial Surgery, Dayananda Sagar College of Dental Sciences, Dayananda Sagar Institutions Bengaluru, Karnataka, India

\section{Honey Verma (Corresponding Author)}

Postgraduate Student, Department of Oral and Maxillofacial Surgery Dayananda Sagar College of Dental Sciences, Dayananda Sagar Institutions, Shavige Malleshwara Hills, Kumaraswamy Layout Bengaluru 560078, Karnataka, India, e-mail: honey_verma12@yahoo.co.in

\section{NT Prashanth}

Associate Professor, Department of Oral and Maxillofacial Surgery Dayananda Sagar College of Dental Sciences, Dayananda Sagar Institutions, Bengaluru, Karnataka, India

\section{ES Shobha}

Professor, Department of Oral and Maxillofacial Surgery, Dayananda Sagar College of Dental Sciences, Dayananda Sagar Institutions Bengaluru, Karnataka, India 